\title{
CONICAL FOURIER-BOREL TRANSFORMATIONS FOR HARMONIC FUNCTIONALS ON THE LIE BALL
}

\author{
MITSUO MORIMOTO \\ Department of Mathematics, Sophia University \\ 7-1, Kioicho, Chiyoda-ku, Tokyo, 102 Japan \\ E-mail: morimoto@mm.sophia.ac.jp \\ KEIKO FUJITA \\ Department of Mathematics, Sophia University \\ 7-1, Kioicho, Chiyoda-ku, Tokyo, 102 Japan \\ E-mail:keiko@mm.sophia.ac.jp
}

\begin{abstract}
Let $L(z)$ be the Lie norm on $\tilde{\mathbb{E}}=\mathbb{C}^{n+1}$ and $L^{*}(z)$ the dual Lie norm. We denote by $\mathcal{O}_{\Delta}(\tilde{B}(R))$ the space of complex harmonic functions on the open Lie ball $\tilde{B}(R)$ and by $\operatorname{Exp}_{\Delta}\left(\tilde{\mathbb{E}} ;\left(A, L^{*}\right)\right)$ the space of entire harmonic functions of exponential type $\left(A, L^{*}\right)$. A continuous linear functional on these spaces will be called a harmonic functional or an entire harmonic functional. We shall study the conical Fourier-Borel transformations on the spaces of harmonic functionals or entire harmonic functionals.
\end{abstract}

\section{Introduction. Let}

$$
K_{\nu}(r)=\int_{0}^{\infty} \exp (-r \cosh t) \cosh \nu t d t, \quad 0<r<\infty,
$$

be the modified Bessel function of degree $\nu \in \mathbb{R}$. K. Ii ([1]) and R. Wada ([5]) introduced the function

$$
\rho_{n}(r)= \begin{cases}\sum_{\ell=0}^{(n-1) / 2} a_{n, \ell} r^{\ell+1} K_{\ell}(r), & \text { if } n \text { is odd, } \\ \sum_{\ell=0}^{n / 2} a_{n, \ell} r^{\ell+1 / 2} K_{\ell-1 / 2}(r), & \text { if } n \text { is even, }\end{cases}
$$

1991 Mathematics Subject Classification: Primary 46F15; Secondary 32A25.

The paper is in final form and no version of it will be published elsewhere. 
where the constants $a_{n, \ell}, \ell=0,1,2, \cdots,[n / 2]$, are determined uniquely by

$$
\int_{0}^{\infty} r^{2 k+n-1} \rho_{n}(r) d r=\frac{N(k, n) k ! \Gamma(k+(n+1) / 2) 2^{2 k}}{\Gamma((n+1) / 2)}=C(k, n)
$$

for $k=0,1,2, \cdots$. If $n$ is even, there is a polynomial $P_{n / 2}(r)$ of degree $n / 2$ such that $\rho_{n}(r)=P_{n / 2}(r) e^{-r}$; if $n$ is odd, there is a polynomial $P_{(n-1) / 2}(r)$ of degree $(n-1) / 2$ such that $\left|\rho_{n}(r)\right| \leq \sqrt{r} P_{(n-1) / 2}(r) e^{-r}$. (See [5] for the details.) We shall call the function $\rho_{n}$ the Ii-Wada function.

Let $\mathbb{E}=\mathbb{R}^{n+1}$ and $S=S^{n}$ be the unit sphere. For $n=2,4,6, \cdots, \mathrm{K}$. Ii considered the integral transformation

$$
\mathcal{F}: f \mapsto \hat{f}(z)=\int_{S} \exp (z \cdot \omega) f(\omega) d S(\omega)
$$

for $z \in \tilde{M}$, where $\tilde{M}=\left\{z \in \tilde{\mathbb{E}} ; z^{2} \equiv z_{1}^{2}+z_{2}^{2}+\cdots+z_{n+1}^{2}=0\right\}$ is the complex light cone. He constructed a "Plancherel" measure $d \tilde{M}$ on $\tilde{M}$ using $\rho_{n}$ and proved that $\mathcal{F}$ is a unitary transformation of $L^{2}(S)$ onto the Hilbert space

$$
L^{2} \mathcal{O}(\tilde{M})=\left\{f \in \mathcal{O}(\tilde{M}) ; \int_{\tilde{M}}|f(\zeta)|^{2} d \tilde{M}(\zeta)<\infty\right\} .
$$

R. Wada proved K. Ii's results for any integer $n \geq 2$ and considered $\mathcal{F}$ for analytic functionals on the complex sphere $\tilde{S}=\left\{z \in \tilde{\mathbb{E}} ; z^{2}=1\right\}$.

In the sequel, we fix a complex number $\lambda \neq 0$. In [3] we studied the growth behavior of homogeneous expansion of holomorphic functions and analytic functionals on $\tilde{M}$ and proved that the Fourier-Borel transformation $\mathcal{F}_{\lambda}$ establishes the following topological linear isomorphisms :

$$
\begin{array}{ll}
\mathcal{F}_{\lambda}: \mathcal{O}^{\prime}(\tilde{M}(R)) \stackrel{\sim}{\longrightarrow} \operatorname{Exp}_{\Delta}\left(\tilde{\mathrm{E}} ;\left[A, L^{*}\right]\right), & \text { where } A=|\lambda| R, \text { and } \\
\mathcal{F}_{\lambda}: \operatorname{Exp}^{\prime}\left(\tilde{M} ;\left(A, L^{*}\right)\right) \stackrel{\sim}{\longrightarrow} \mathcal{O}_{\Delta}(\tilde{B}[R]), & \text { where } R=A /|\lambda| .
\end{array}
$$

We shall show that the conical Fourier-Borel transformation $\mathcal{F}_{\lambda}^{\Delta}$ establishes the following topological linear isomorphisms :

$$
\begin{array}{ll}
\mathcal{F}_{\lambda}^{\Delta}: \mathcal{O}_{\Delta}^{\prime}(\tilde{B}(R)) \stackrel{\sim}{\longrightarrow} \operatorname{Exp}\left(\tilde{M} ;\left[A, L^{*}\right]\right), & \text { where } A=|\lambda| R, \text { and } \\
\mathcal{F}_{\lambda}^{\Delta}: \operatorname{Exp}_{\Delta}^{\prime}\left(\tilde{\mathbb{E}} ;\left(A, L^{*}\right)\right) \stackrel{\sim}{\longrightarrow} \mathcal{O}(\tilde{M}[R]), & \text { where } R=A /|\lambda| .
\end{array}
$$

By means of the Plancherel measure on $\tilde{M}$, we shall construct a duality bilinear form on $\operatorname{Exp}\left(\tilde{M} ;\left(A, L^{*}\right)\right) \times \operatorname{Exp}\left(\tilde{M} ;\left[1 / A, L^{*}\right]\right)$ (Theorem 17). The first main theorem (Theorem 18) asserts that this duality is related with the Poisson duality (Theorem 5) of $\mathcal{O}_{\Delta}(\tilde{B}(R))$ and $\mathcal{O}_{\Delta}(\tilde{B}[1 / R])$ via $(5)$ and $(4)$.

Similarly, we shall construct the Plancherel measure on $\mathbb{E}$ and a duality bilinear form on $\operatorname{Exp}_{\Delta}\left(\tilde{\mathbb{E}} ;\left(A, L^{*}\right)\right) \times \operatorname{Exp}_{\Delta}\left(\tilde{\mathbb{E}} ;\left[1 / A, L^{*}\right]\right)$ (Theorem 25). The second main theorem (Theorem 26) asserts that this duality is related with the Cauchy duality (Theorem 11) of $\mathcal{O}(\tilde{M}(R))$ and $\mathcal{O}(\tilde{M}[1 / R])$ via (3) and (6).

The second named author discovered the integral formulas of the inverse mapping of these four transformations. Later, through our discussion her results have been rearranged as stated in our main theorems. 
2. Complex harmonic functions on $\tilde{B}(R)$. We recall some notations. We refer the reader to [4] and [2] for general background of this section.

Let $\|x\|$ be the Euclidean norm on $\mathbb{E}=\mathbb{R}^{n+1}, n \geq 2$. The cross norm $L(z)$ on $\tilde{\mathbb{E}}=\mathbb{C}^{n+1}$ corresponding to $\|x\|$ is the Lie norm defined by

$$
L(z)=L(x+i y)=\left[\|x\|^{2}+\|y\|^{2}+2 \sqrt{\|x\|^{2}\|y\|^{2}-(x \cdot y)^{2}}\right]^{1 / 2},
$$

where $z=x+i y, x, y \in \mathbb{E}$ and $x \cdot y=x_{1} y_{1}+x_{2} y_{2}+\cdots+x_{n+1} y_{n+1}$. We denote by $L^{*}(z)$ the dual Lie norm:

$$
\begin{aligned}
L^{*}(z) & =\sup \{|z \cdot \zeta| ; L(\zeta) \leq 1\} \\
& =\frac{1}{\sqrt{2}}\left[\|x\|^{2}+\|y\|^{2}+\sqrt{\left(\|x\|^{2}-\|y\|^{2}\right)^{2}+4(x \cdot y)^{2}}\right]^{1 / 2} .
\end{aligned}
$$

The open and the closed Lie balls of radius $R$ with center at 0 are defined by

$$
\begin{aligned}
\tilde{B}(R) & =\{z \in \tilde{\mathbb{E}} ; L(z)<R\}, & & 0<R \leq \infty, \\
\tilde{B}[R] & =\{z \in \tilde{\mathbb{E}} ; L(z) \leq R\}, & & 0 \leq R<\infty .
\end{aligned}
$$

Let $\mathcal{O}(\tilde{B}(R))$ be the space of holomorphic functions on $\tilde{B}(R)$ with the topology of uniform convergence on compact sets. We denote by

$$
\mathcal{O}_{\Delta}(\tilde{B}(R))=\left\{f \in \mathcal{O}(\tilde{B}(R)) ; \Delta_{z} f(z)=0\right\}
$$

the space of complex harmonic functions on $\tilde{B}(R)$, where

$$
\Delta_{z} f(z)=\left(\partial^{2} / \partial z_{1}^{2}+\partial^{2} / \partial z_{2}^{2}+\cdots+\partial^{2} / \partial z_{n+1}^{2}\right) f(z) .
$$

For $0 \leq R<\infty$ we put

$$
\begin{aligned}
\mathcal{O}(\tilde{B}[R]) & =\text { ind } \lim \left\{\mathcal{O}\left(\tilde{B}\left(R^{\prime}\right)\right) ; R^{\prime}>R\right\}, \\
\mathcal{O}_{\Delta}(\tilde{B}[R]) & =\text { ind } \lim \left\{\mathcal{O}_{\Delta}\left(\tilde{B}\left(R^{\prime}\right)\right) ; R^{\prime}>R\right\}
\end{aligned}
$$

A continuous linear functional on $\mathcal{O}_{\Delta}(\tilde{B}(R))$ (resp., $\mathcal{O}_{\Delta}(\tilde{B}[R])$ ) is called a harmonic functional on $\tilde{B}(R)$ (resp., $\tilde{B}[R])$. We denote by $\mathcal{O}_{\Delta}^{\prime}(\tilde{B}(R))$ (resp., $\left.\mathcal{O}_{\Delta}^{\prime}(\tilde{B}[R])\right)$ the dual space of $\mathcal{O}_{\Delta}(\tilde{B}(R))$ (resp., $\left.\mathcal{O}_{\Delta}(\tilde{B}[R])\right)$.

We denote by $\mathcal{P}^{k}(\tilde{\mathbb{E}})$ the space of $k$-homogeneous polynomials of $n+1$ variables with complex coefficients. Denote by

$$
\mathcal{P}_{\Delta}^{k}(\tilde{\mathbb{E}})=\left\{f \in \mathcal{P}^{k}(\tilde{\mathbb{E}}) ; \Delta_{z} f(z)=0\right\}
$$

the space of complex harmonic polynomials of degree $k$. The dimension of $\mathcal{P}_{\Delta}^{k}(\tilde{\mathbb{E}})$ is given by $N(k, n)=(2 k+n-1)(k+n-2) ! /(k !(n-1) !)$. Let $P_{k, n}(t)$ be the Legendre polynomial of degree $k$ and of dimension $n+1$. We put

$$
\tilde{P}_{k, n}(z, w)=\left(\sqrt{z^{2}}\right)^{k}\left(\sqrt{w^{2}}\right)^{k} P_{k, n}\left(\left(z / \sqrt{z^{2}}\right) \cdot\left(w / \sqrt{w^{2}}\right)\right),
$$

where $z^{2}=z_{1}^{2}+z_{2}^{2}+\cdots+z_{n+1}^{2}$. Then $\tilde{P}_{k, n}(z, w)$ is a symmetric polynomial in $z$ and $w$, which satisfies $\Delta_{z} \tilde{P}_{k, n}(z, w)=\Delta_{w} \tilde{P}_{k, n}(z, w)=0$ and $\left|\tilde{P}_{k, n}(z, w)\right| \leq L(z)^{k} L(w)^{k}$.

Let $S=\{x \in \mathbb{E} ;\|x\|=1\}$ be the sphere of dimension $n$ and $d S$ the normalized invariant measure on $S$.

Lemma 1. Let $f_{k} \in \mathcal{P}_{\Delta}^{k}(\tilde{\mathbb{E}})$ and $f_{j} \in \mathcal{P}_{\Delta}^{j}(\tilde{\mathbb{E}})$. Then we have

1) $\int_{S} f_{k}(\omega) f_{j}(\omega) d S(\omega)=0, \quad k \neq j$; 
2) $\quad f_{k}(z)=N(k, n) \int_{S} f_{k}(\omega) \tilde{P}_{k, n}(z, \omega) d S(\omega), z \in \tilde{\mathbb{E}}$.

The Poisson kernel $K_{1}(z, w)$ is defined by

$$
K_{1}(z, w)=\frac{1-z^{2} w^{2}}{\left(1+z^{2} w^{2}-2 z \cdot w\right)^{(n+1) / 2}}=\sum_{k=0}^{\infty} N(k, n) \tilde{P}_{k, n}(z, w) .
$$

Then $K_{1}(z, w)$ is a symmetric holomorphic function on

$$
\{(z, w) \in \tilde{\mathbb{E}} \times \tilde{\mathbb{E}} ; L(z) L(w)<1\}
$$

and satisfies $\Delta_{z} K_{1}(z, w)=\Delta_{w} K_{1}(z, w)=0$.

Theorem 2. (Poisson integral formula) Let $0<\rho<R$ and $f \in \mathcal{O}_{\Delta}(\tilde{B}(R))$. Then we have

$$
f(z)=\int_{S} f(\rho \omega) K_{1}(z, \omega / \rho) d S(\omega), \quad z \in \tilde{B}(\rho) .
$$

Corollary 3. Define the k-harmonic component $f_{k}$ of $f \in \mathcal{O}_{\Delta}(\tilde{B}(R))$ by

$$
f_{k}(z)=N(k, n) \int_{S} f(\rho \omega) \tilde{P}_{k, n}(z, \omega / \rho) d S(\omega), 0<\rho<R .
$$

Then $f_{k} \in \mathcal{P}_{\Delta}^{k}(\tilde{\mathbb{E}})$ and we have $f(z)=\sum_{k=0}^{\infty} f_{k}(z)$, where the convergence is uniform on compact sets in $\tilde{B}(R)$.

Lemma 4. Let $f \in \mathcal{O}_{\Delta}(\tilde{B}(R))$ and $g \in \mathcal{O}_{\Delta}(\tilde{B}[1 / R])$. The bilinear form

$$
\langle f, g\rangle_{S}=\int_{S} f(\rho \omega) g(\omega / \rho) d S(\omega)
$$

is well-defined, where $0<\rho<R$ is sufficiently close to $R$. (8) is separately continuous.

Proof. Take $R^{\prime}<R$ such that $g \in \mathcal{O}_{\Delta}\left(\tilde{B}\left(1 / R^{\prime}\right)\right)$. If $R^{\prime}<\rho<R$, then Corollary 3 and Lemma 1 imply

$$
\begin{aligned}
\int_{S} f(\rho \omega) g(\omega / \rho) d S(\omega) & =\int_{S} \sum_{k=0}^{\infty} f_{k}(\rho \omega) \sum_{\ell=0}^{\infty} f_{\ell}(\omega / \rho) d S(\omega) \\
& =\sum_{k=0}^{\infty} \int_{S} f_{k}(\omega) g_{k}(\omega) d S(\omega)
\end{aligned}
$$

Therefore, the right-hand side of (8) does not depend on $\rho$ and the bilinear form is well-defined.

We claim that (8) establishes the duality of these spaces.

Let $T \in \mathcal{O}_{\Delta}^{\prime}(\tilde{B}(R))$. If $w \in \tilde{B}[1 / R]$, then the function $K_{1}(\cdot, w)$ belongs to $\mathcal{O}_{\Delta}(\tilde{B}(R))$. We define the Poisson transform $\mathcal{P} T$ of $T$ by

$$
\mathcal{P} T(w)=\left\langle T_{z}, K_{1}(z, w)\right\rangle, \quad w \in \tilde{B}[1 / R] .
$$

We call the mapping $\mathcal{P}: T \longmapsto \mathcal{P} T$ the Poisson transformation.

THEOREM 5. The Poisson transformation $\mathcal{P}$ establishes the following topological linear isomorphisms:

$$
\begin{aligned}
& \mathcal{P}: \mathcal{O}_{\Delta}^{\prime}(\tilde{B}(R)) \stackrel{\sim}{\longrightarrow} \mathcal{O}_{\Delta}(\tilde{B}[1 / R]), \quad 0<R \leq \infty, \\
& \mathcal{P}: \mathcal{O}_{\Delta}^{\prime}(\tilde{B}[R]) \stackrel{\sim}{\longrightarrow} \mathcal{O}_{\Delta}(\tilde{B}(1 / R)), \quad 0 \leq R<\infty .
\end{aligned}
$$


We have $\langle T, f\rangle=\langle f, \mathcal{P} T\rangle_{S}$ for $T \in \mathcal{O}_{\Delta}^{\prime}(\tilde{B}(R))$ and $f \in \mathcal{O}_{\Delta}(\tilde{B}(R))$ or for $T \in \mathcal{O}_{\Delta}^{\prime}(\tilde{B}[R])$ and $f \in \mathcal{O}_{\Delta}(\tilde{B}[R])$.

Pro of. We prove only (i). Let $T \in \mathcal{O}_{\Delta}^{\prime}(\tilde{B}(R))$. By the Hahn-Banach theorem, there is a Radon measure $\mu$ with supp $\mu \subset \tilde{B}\left(R^{\prime}\right), R^{\prime}<R$, such that $\langle T, f\rangle=\int_{\tilde{B}\left(R^{\prime}\right)} f(z) d \mu(z)$ for $f \in \mathcal{O}_{\Delta}(\tilde{B}(R))$. Especially, we have

$$
\mathcal{P} T(w)=\int_{\tilde{B}\left(R^{\prime}\right)} K_{1}(z, w) d \mu(z) .
$$

Therefore, $\mathcal{P} T$ can be extended holomorphically to $\tilde{B}\left(1 / R^{\prime}\right)$ and satisfies

$$
\Delta_{w} \mathcal{P} T(w)=0
$$

that is, $\mathcal{P} T \in \mathcal{O}_{\Delta}(\tilde{B}[1 / R])$.

Let $f \in \mathcal{O}_{\Delta}(\tilde{B}(R))$. If $R^{\prime}<\rho<R$, then Theorem 2 and the Fubini theorem imply

$$
\begin{aligned}
\langle T, f\rangle & =\int_{\tilde{B}\left(R^{\prime}\right)} \int_{S} f(\rho \omega) K_{1}(z, \omega / \rho) d S(\omega) d \mu(z) \\
& =\int_{S} f(\rho \omega) \int_{\tilde{B}\left(R^{\prime}\right)} K_{1}(z, \omega / \rho) d \mu(z) d S(\omega) \\
& =\int_{S} f(\rho \omega) \mathcal{P} T(\omega / \rho) d S(\omega)=\langle f, \mathcal{P} T\rangle_{S}
\end{aligned}
$$

Thus, $\mathcal{P}$ is injective. The continuity of $\mathcal{P}$ is clear.

Conversely, let $F \in \mathcal{O}_{\Delta}(\tilde{B}[1 / R])$. Then there is $R^{\prime}$ with $0<R^{\prime}<R$ such that $F \in \mathcal{O}_{\Delta}\left(\tilde{B}\left(1 / R^{\prime}\right)\right)$. Take $\rho$ with $R^{\prime}<\rho<R$ and define $T_{F} \in \mathcal{O}_{\Delta}^{\prime}(\tilde{B}(R))$ by

$$
\left\langle T_{F}, f\right\rangle=\int_{S} f(\rho \omega) F(\omega / \rho) d S(\omega)=\langle f, F\rangle_{S}, \quad f \in \mathcal{O}_{\Delta}(\tilde{B}(R)) .
$$

Then Theorem 2 implies

$$
\begin{aligned}
\mathcal{P} T_{F}(w) & =\left\langle\left(T_{F}\right)_{z}, K_{1}(z, w)\right\rangle \\
& =\int_{S} K_{1}(\rho \omega, w) F(\omega / \rho) d S(\omega)=F(w), \quad w \in \tilde{B}(1 / \rho) .
\end{aligned}
$$

Thus $\mathcal{P}$ is surjective. The continuity of $\mathcal{P}^{-1}: F \mapsto T_{F}$ is clear.

3. Holomorphic functions on $\tilde{M}(R)$. Let $\tilde{M}=\left\{\zeta \in \tilde{\mathbb{E}} ; \zeta^{2}=0\right\}$ be the complex light cone. We put

$$
\tilde{M}(R)=\tilde{M} \cap \tilde{B}(R), \quad \tilde{M}[R]=\tilde{M} \cap \tilde{B}[R] .
$$

We denote by $\mathcal{O}(\tilde{M}(R))$ the space of holomorphic functions on $\tilde{M}(R)$ and put $\mathcal{O}(\tilde{M}[R])=$ ind $\lim \left\{\mathcal{O}\left(\tilde{M}\left(R^{\prime}\right)\right) ; R^{\prime}>R\right\}$. A continuous linear functional on $\mathcal{O}(\tilde{M}(R)$ ) (resp., $\mathcal{O}(\tilde{M}[R])$ ) is called an analytic functional on $\tilde{M}(R)$ (resp., $\tilde{M}[R])$. We denote by $\mathcal{O}^{\prime}(\tilde{M}(R))$ (resp., $\left.\mathcal{O}^{\prime}(\tilde{M}[R])\right)$ the dual space of $\mathcal{O}(\tilde{M}(R))$ (resp., $\left.\mathcal{O}(\tilde{M}[R])\right)$.

We denote the space of $k$-homogeneous polynomials on $\tilde{M}$ by $\mathcal{P}^{k}(\tilde{M})=\left\{\left.P\right|_{\tilde{M}} ; P \in\right.$ $\left.\mathcal{P}^{k}(\tilde{\mathbb{E}})\right\}$. Note that, if $\xi$ or $\zeta \in \tilde{M}$, then $\tilde{P}_{k, n}(\xi, \zeta)=\gamma(k, n)(\xi \cdot \zeta)^{k}$, where $\gamma(k, n)$ is the coefficient of the highest power of $P_{k, n}(t)$ :

$$
\gamma(k, n)=\Gamma(k+(n+1) / 2) 2^{k} /(N(k, n) \Gamma((n+1) / 2) k !) .
$$


Let $M=\{\zeta \in \tilde{M} ; L(\zeta)=1\}$. Then $M$ is isomorphic to the cotangential sphere bundle over $S$ and $M \stackrel{\sim}{=} \mathrm{O}(n+1) / \mathrm{O}(n-1)$. We denote by $d M$ the normalized invariant measure on $M$.

LEMMA 6. ([5, Lemma 1.3]) Let $f_{k} \in \mathcal{P}_{\Delta}^{k}(\tilde{\mathbb{E}})$ and $f_{j} \in \mathcal{P}_{\Delta}^{j}(\tilde{\mathbb{E}})$.

1) $\int_{M} f_{k}(\zeta) f_{j}(\bar{\zeta}) d M(\zeta)=0, \quad k \neq j$

2) $\quad f_{k}(z)=2^{k} N(k, n) \int_{M} f_{k}(\zeta)(z \cdot \bar{\zeta})^{k} d M(\zeta), \quad z \in \tilde{\mathbb{E}}$.

The Cauchy kernel $K_{0}(\xi, \zeta)$ is defined by

$$
K_{0}(\xi, \zeta)=\frac{1+2 \xi \cdot \zeta}{(1-2 \xi \cdot \zeta)^{n}}=\sum_{k=0}^{\infty} 2^{k} N(k, n)(\xi \cdot \zeta)^{k} .
$$

Then $K_{0}(\xi, \zeta)$ is a symmetric holomorphic function on

$$
\left\{(\xi, \zeta) \in \tilde{\mathbb{E}} \times \tilde{\mathbb{E}} ; L(\xi) L^{*}(\zeta)<1 / 2 \text { or } L^{*}(\xi) L(\zeta)<1 / 2\right\} .
$$

Since

$$
2 L^{*}(\zeta)=L(\zeta) \text { for } \zeta \in \tilde{M}
$$

if $\zeta \in \tilde{M}$, then $K_{0}(\cdot, \zeta)$ is a holomorphic function on $\{z \in \tilde{\mathbb{E}} ; L(z) L(\zeta)<1\}$ and satisfies $\Delta_{z} K_{0}(z, \zeta)=0$.

TheOREM 7. (Cauchy integral formula for $\mathcal{O}_{\Delta}(\tilde{B}(R))$ ) Let $0<\rho<R$ and $f \in$ $\mathcal{O}_{\Delta}(\tilde{B}(R))$. Then we have

$$
f(z)=\int_{M} f(\rho \zeta) K_{0}(z, \bar{\zeta} / \rho) d M(\zeta), \quad z \in \tilde{B}(\rho)
$$

For $f \in \mathcal{O}_{\Delta}(\tilde{B}(R))$ the $k$-harmonic component of $f$ is also given by

$$
f_{k}(z)=2^{k} N(k, n) \int_{M} f(\rho \zeta)(z \cdot \bar{\zeta} / \rho)^{k} d M(\zeta), \quad z \in \tilde{\mathbb{E}},
$$

where $0<\rho<R$. It is known $([7])$ that the restriction mapping $\left.f \mapsto f\right|_{\tilde{M}(R)}$ is a topological linear isomorphism of $\mathcal{O}_{\Delta}(\tilde{B}(R))$ onto $\mathcal{O}(\tilde{M}(R))$. The inverse mapping is given by the right-hand side of (11). $f \in \mathcal{O}_{\Delta}(\tilde{B}(R))$ is called the harmonic extension of $\left.f\right|_{\tilde{M}(R)}$.

We shall consider functions on $\tilde{M}$. If we consider $K_{0}(\xi, \zeta)$ as a function on $\tilde{M} \times \tilde{M}$, it is a symmetric holomorphic function on $\{(\xi, \zeta) \in \tilde{M} \times \tilde{M} ; L(\xi) L(\zeta)<1\}$.

Theorem 8. (Cauchy integral formula on $\tilde{M}$ ) Let $0<\rho<R$ and $f \in \mathcal{O}(\tilde{M}(R))$. Then we have

$$
f(\xi)=\int_{M} f(\rho \zeta) K_{0}(\xi, \bar{\zeta} / \rho) d M(\zeta), \quad \xi \in \tilde{M}(\rho)
$$

Corollary 9. ([3, Theorem 5]) Define the $k$-homogeneous component $f_{k}$ of $f \in$ $\mathcal{O}(\tilde{M}(R))$ by

$$
f_{k}(\xi)=2^{k} N(k, n) \int_{M} f(\rho \zeta)(\xi \cdot \bar{\zeta} / \rho)^{k} d M(\zeta), \quad 0<\rho<R .
$$

Then $f_{k} \in \mathcal{P}^{k}(\tilde{M})$ and we have $f(\xi)=\sum_{k=0}^{\infty} f_{k}(\xi)$, where the convergence is uniform on compact sets in $\tilde{M}(R)$. 
Lemma 10. Let $f \in \mathcal{O}(\tilde{M}(R))$ and $g \in \mathcal{O}(\tilde{M}[1 / R])$. The bilinear form

$$
\langle f, g\rangle_{M}=\int_{M} f(\rho \zeta) g(\bar{\zeta} / \rho) d M(\zeta)
$$

is well-defined, where $0<\rho<R$ is sufficiently close to $R$. (14) is separately continuous.

Proof. Take $R^{\prime}<R$ such that $g \in \mathcal{O}\left(\tilde{M}\left(1 / R^{\prime}\right)\right)$. If $R^{\prime}<\rho<R$, then Corollary 9 and Lemma 6 imply

$$
\begin{aligned}
\int_{M} f(\rho \zeta) g(\bar{\zeta} / \rho) d M(\zeta) & =\int_{M} \sum_{k=0}^{\infty} f_{k}(\rho \zeta) \sum_{\ell=0}^{\infty} g_{\ell}(\bar{\zeta} / \rho) d M(\zeta) \\
& =\sum_{k=0}^{\infty} \int_{M} f_{k}(\zeta) g_{k}(\bar{\zeta}) d M(\zeta) .
\end{aligned}
$$

Therefore, the right-hand side of (14) does not depend on $\rho$ and the bilinear form is well-defined.

We claim that (14) establishes the duality of these spaces.

Let $T \in \mathcal{O}^{\prime}(\tilde{M}(R))$. If $\zeta \in \tilde{M}[1 / R]$, then the function $K_{0}(\cdot, \zeta)$ belongs to $\mathcal{O}(\tilde{M}(R))$. We define the Cauchy transform $\mathcal{C} T$ of $T$ by

$$
\mathcal{C} T(\zeta)=\left\langle T_{\xi}, K_{0}(\xi, \zeta)\right\rangle, \quad \zeta \in \tilde{M}[1 / R]
$$

We call the mapping $\mathcal{C}: T \longmapsto \mathcal{C} T$ the Cauchy transformation.

Because $\mathcal{C} T$ can be continued holomorphically to a neighborhoood of $\tilde{M}[1 / R]$, the Cauchy transformation is a continuous linear mapping of $\mathcal{O}^{\prime}(\tilde{M}(R))$ into $\mathcal{O}(\tilde{M}[1 / R])$. We proved the following theorem in [3].

THEOREM 11. The Cauchy transformation $\mathcal{C}$ establishes the following topological linear isomorphisms:

$$
\begin{array}{lll}
\mathcal{C}: & \mathcal{O}^{\prime}(\tilde{M}(R)) \stackrel{\sim}{\longrightarrow} \mathcal{O}(\tilde{M}[1 / R]), & 0<R \leq \infty, \\
\mathcal{C}: & \mathcal{O}^{\prime}(\tilde{M}[R]) \stackrel{\sim}{\longrightarrow} \mathcal{O}(\tilde{M}(1 / R)), & 0 \leq R<\infty .
\end{array}
$$

We have $\langle T, f\rangle=\langle f, \mathcal{C} T\rangle_{M}$ for $T \in \mathcal{O}^{\prime}(\tilde{M}(R))$ and $f \in \mathcal{O}(\tilde{M}(R))$ or for $T \in \mathcal{O}^{\prime}(\tilde{M}[R])$ and $f \in \mathcal{O}(\tilde{M}[R])$.

Note that the right-hand side of $(15)$ is defined for $\zeta \in \tilde{B}[1 / R]$ and satisfies $\Delta_{\zeta} \mathcal{C} T(\zeta)=$ 0 . Hence, we can consider the Cauchy transformation as a topological linear isomorphism: $\mathcal{O}^{\prime}(\tilde{M}(R)) \stackrel{\sim}{\longrightarrow} \mathcal{O}_{\Delta}(\tilde{B}[1 / R]) .($ See $[3$, Theorem 9$]$.

4. Conical Fourier-Borel transformation. If $\zeta \in \tilde{M}$, then the function $\exp (\lambda \zeta \cdot z)$ is entire harmonic in $z$. Therefore, for $T \in \mathcal{O}_{\Delta}^{\prime}(\tilde{B}(R))$, the value $\left\langle T_{z}, \exp (\lambda \zeta \cdot z)\right\rangle$ is welldefined, which we denote by

$$
\mathcal{F}_{\lambda}^{\Delta} T(\zeta)=\left\langle T_{z}, \exp (\lambda \zeta \cdot z)\right\rangle, \quad \zeta \in \tilde{M}
$$

We call $\mathcal{F}_{\lambda}^{\Delta} T$ the conical Fourier-Borel transform of $T$. 
By the Hahn-Banach theorem, there is a Radon measure $\mu$ on $\tilde{B}(R)$ with supp $\mu \subset$ $\tilde{B}\left[R^{\prime}\right], R^{\prime}<R$, such that

$$
\mathcal{F}_{\lambda}^{\Delta} T(\zeta)=\int_{\tilde{B}\left[R^{\prime}\right]} \exp (\lambda \zeta \cdot z) d \mu(z)
$$

Therefore, we have

$$
\begin{aligned}
\left|\mathcal{F}_{\lambda}^{\Delta} T(\zeta)\right| & \leq\|\mu\| \sup \left\{|\exp (\lambda \zeta \cdot z)| ; z \in \tilde{B}\left[R^{\prime}\right]\right\} \\
& =\|\mu\| \exp \left(|\lambda| R^{\prime} L^{*}(\zeta)\right) .
\end{aligned}
$$

Thus, $\mathcal{F}_{\lambda}^{\Delta} T$ is an entire function on $\tilde{M}$ (that is, a holomorphic function on $\tilde{M}$ ) of exponential type. To describe the image under $\mathcal{F}_{\lambda}^{\Delta}$, we introduce some notations.

Let $N$ be the Lie norm $L$ or the dual Lie norm $L^{*}$. For $0<A \leq \infty$ we denote by

$\operatorname{Exp}(\tilde{M} ;[A, N])=\left\{f \in \mathcal{O}(\tilde{M}) ;\right.$ there is $A^{\prime}<A$ such that

$$
\left.\sup \left\{|f(\zeta)| \exp \left(-A^{\prime} N(\zeta)\right) ; \zeta \in \tilde{M}\right\}<\infty\right\}
$$

the space of entire functions on $\tilde{M}$ of exponential type $[A, N]$. Furthermore, for $0 \leq A<$ $\infty$ we put

$$
\begin{aligned}
\operatorname{Exp}(\tilde{M} ;(A, N)) & =\left\{f \in \mathcal{O}(\tilde{M}) ; \text { for all } A^{\prime}>A\right. \\
& \left.\sup \left\{|f(\zeta)| \exp \left(-A^{\prime} N(\zeta)\right) ; \zeta \in \tilde{M}\right\}<\infty\right\} .
\end{aligned}
$$

Using these notations, we can state the following

LEMma 12. The conical Fourier-Borel transformation $\mathcal{F}_{\lambda}^{\Delta}$ establishes the following continuous linear mappings:

$$
\begin{array}{lll}
\mathcal{F}_{\lambda}^{\Delta}: & \mathcal{O}_{\Delta}^{\prime}(\tilde{B}(R)) \longrightarrow \operatorname{Exp}\left(\tilde{M} ;\left[|\lambda| R, L^{*}\right]\right), & 0<R \leq \infty, \\
\mathcal{F}_{\lambda}^{\Delta}: & \mathcal{O}_{\Delta}^{\prime}(\tilde{B}[R]) \longrightarrow \operatorname{Exp}\left(\tilde{M} ;\left(|\lambda| R, L^{*}\right)\right), & 0 \leq R<\infty .
\end{array}
$$

We shall show that the transformations $\mathcal{F}_{\lambda}^{\Delta}$ are topological linear isomorphisms (Theorem 18).

5. The Plancherel measure on $\tilde{M}$. K. Ii ([1]) defined the Plancherel measure $d \tilde{M}$ on $\tilde{M}$ by

$$
\int_{\tilde{M}} f(\zeta) d \tilde{M}(\zeta)=\int_{0}^{\infty} \int_{M} f\left(r \zeta^{\prime}\right) d M\left(\zeta^{\prime}\right) r^{n-1} \rho_{n}(r) d r, \quad \zeta=r \zeta^{\prime}
$$

where $\rho_{n}$ is the Ii-Wada function defined by (1). By means of the Plancherel measure on $\tilde{M}$, we can define a duality bilinear form on

$$
\operatorname{Exp}\left(\tilde{M} ;\left(A, L^{*}\right)\right) \times \operatorname{Exp}\left(\tilde{M} ;\left[1 / A, L^{*}\right]\right) .
$$

Lemma 13. Let $A \geq 0, f \in \operatorname{Exp}\left(\tilde{M} ;\left(A, L^{*}\right)\right)$ and $g \in \operatorname{Exp}\left(\tilde{M} ;\left[1 / A, L^{*}\right]\right)$.

1) The integral

$$
\int_{\tilde{M}} f(s \zeta) g(\bar{\zeta} / s) d \tilde{M}(\zeta)
$$

converges absolutely for $s \in \mathbb{C}$ with $0<|s|<1 / A$ sufficiently close to $1 / A$. 
2) The integral (16) is independent of s. The bilinear form

$$
\langle\langle f, g\rangle\rangle_{\tilde{M}}=\int_{\tilde{M}} f(s \zeta) g(\bar{\zeta} / s) d \tilde{M}(\zeta)
$$

is well-defined and separately continuous. We have

$$
\langle\langle f, g\rangle\rangle_{M}=\sum_{k=0}^{\infty} C(k, n) \int_{M} f_{k}\left(\zeta^{\prime}\right) g_{k}\left(\bar{\zeta}^{\prime}\right) d M\left(\zeta^{\prime}\right),
$$

where $f_{k}$ and $g_{k}$ are the $k$-homogeneous components of $f$ and $g$ respectively, and $C(k, n)$ are the constants given by (2).

Proof. 1) Take $A^{\prime}>A$ and $C^{\prime} \geq 0$ such that

$$
|g(\bar{\zeta} / s)| \leq C^{\prime} \exp \left(1 /\left(|s| A^{\prime}\right) L^{*}(\zeta)\right), \quad \zeta \in \tilde{M}
$$

For any $A^{\prime \prime}$ with $A^{\prime}>A^{\prime \prime}>A$ there is $C^{\prime \prime} \geq 0$ such that

$$
|f(s \zeta)| \leq C^{\prime \prime} \exp \left(|s| A^{\prime \prime} L^{*}(\zeta)\right), \quad \zeta \in \tilde{M} .
$$

Therefore, from (10) for $|s|=1 / A^{\prime \prime}$ there is $C \geq 0$ such that

$$
|f(s \zeta) g(\bar{\zeta} / s)| \leq C \exp \left(\left(1+A^{\prime \prime} / A^{\prime}\right) L(\zeta) / 2\right) .
$$

Because $\rho_{n}(r)$ is of exponential type -1 , we have 1).

2) Take $s \in \mathbb{C} \backslash\{0\}$ as in 1$)$. Put $\zeta=r \zeta^{\prime}, r \geq 0, \zeta^{\prime} \in M$. Then by Lemma 6 , Corollary 9 and (2) we have

$$
\begin{aligned}
\int_{\tilde{M}} f(s \zeta) g(\bar{\zeta} / s) d \tilde{M} & =\int_{0}^{\infty} \int_{M} \sum_{k=0}^{\infty} s^{k} r^{k} f_{k}\left(\zeta^{\prime}\right) \sum_{\ell=0}^{\infty} r^{\ell} s^{-\ell} g_{\ell}\left(\bar{\zeta}^{\prime}\right) d M\left(\zeta^{\prime}\right) r^{n-1} \rho_{n}(r) d r \\
& =\int_{0}^{\infty} \sum_{k=0}^{\infty} r^{2 k} \int_{M} f_{k}\left(\zeta^{\prime}\right) g_{k}\left(\bar{\zeta}^{\prime}\right) d M\left(\zeta^{\prime}\right) r^{n-1} \rho_{n}(r) d r \\
& =\sum_{k=0}^{\infty} C(k, n) \int_{M} f_{k}\left(\zeta^{\prime}\right) g_{k}\left(\bar{\zeta}^{\prime}\right) d M\left(\zeta^{\prime}\right) .
\end{aligned}
$$

Lemma 14. For $z, w \in \tilde{\mathbb{E}}$ with $L(z) L(w)<1$ the Poisson kernel $K_{1}(z, w)$ can be represented as follows:

$$
K_{1}(z, w)=\int_{\tilde{M}} \exp (s z \cdot \zeta) \exp (\bar{\zeta} \cdot w / s) d \tilde{M}(\zeta)
$$

where $s \in \mathbb{C}$ is sufficiently close to $1 / L(z)$.

Proof. The convergence of the integral can be proved as in the proof of Lemma 13. 
Now by Lemma $6,(2),(9)$ and (7), we have

$$
\begin{aligned}
\int_{\tilde{M}} \exp (s z \cdot \zeta) \exp (\bar{\zeta} \cdot w / s) d \tilde{M}(\zeta) \\
\quad=\sum_{k=0}^{\infty} C(k, n) \int_{M}\left(\left(z \cdot \zeta^{\prime}\right)^{k} / k !\right)\left(\left(\overline{\zeta^{\prime}} \cdot w\right)^{k} / k !\right) d M\left(\zeta^{\prime}\right) \\
=\sum_{k=0}^{\infty} C(k, n) /\left((k !)^{2} 2^{k} N(k, n) \gamma(k, n)\right) \tilde{P}_{k, n}(z, w) \\
=\sum_{k=0}^{\infty} N(k, n) \tilde{P}_{k, n}(z, w)=K_{1}(z, w)
\end{aligned}
$$

K. Ii ([1]) defined the kernel $E_{0}(\xi, \zeta)$ by

$$
E_{0}(\xi, \zeta)=\int_{S} \exp (\xi \cdot \omega) \exp (\omega \cdot \zeta) d S(\omega),
$$

which we shall call the F-Poisson kernel. By the definition, $E_{0}(\xi, \zeta)$ is a symmetric holomorphic function on $\tilde{\mathbb{E}} \times \tilde{\mathbb{E}}$ and satisfies

$$
\left|E_{0}(\xi, \zeta)\right| \leq \exp \left(L^{*}(\xi)+L^{*}(\zeta)\right), \quad(\xi, \zeta) \in \tilde{\mathbb{E}} \times \tilde{\mathbb{E}}
$$

LEMMA 15. We have

$$
E_{0}(\xi, \zeta)=\sum_{k=0}^{\infty} 2^{k} N(k, n) / C(k, n)(\xi \cdot \zeta)^{k}, \quad \xi, \zeta \in \tilde{M}
$$

Especially, for any $s \in \mathbb{C} \backslash\{0\}$ we have

$$
E_{0}(s \xi, \zeta / s)=E_{0}(\xi, \zeta), \quad \xi, \zeta \in \tilde{M}
$$

Pro of. Let $\xi, \zeta \in \tilde{M}$. Then by Corollary 3, Lemma 1, (2) and (9), we have

$$
\begin{aligned}
E_{0}(\xi, \zeta) & =\sum_{k=0}^{\infty} 1 /(k !)^{2} \int_{S}(\xi \cdot \omega)^{k}(\omega \cdot \zeta)^{k} d S(\omega) \\
& =\sum_{k=0}^{\infty} 1 /\left((k !)^{2} \gamma(k, n) N(k, n)\right)(\xi \cdot \zeta)^{k} \\
& =\sum_{k=0}^{\infty} 2^{k} N(k, n) / C(k, n)(\xi \cdot \zeta)^{k} .
\end{aligned}
$$

The F-Poisson kernel has a reproducing property in the following sense.

Theorem 16. Let $A \geq 0$ and $f \in \operatorname{Exp}\left(\tilde{M} ;\left(A, L^{*}\right)\right)$. For $s \in \mathbb{C}$ with $0<|s|<2 / A$ we have

$$
f(\xi)=\int_{\tilde{M}} f(s \zeta) E_{0}(\xi, \bar{\zeta} / s) d \tilde{M}(\zeta), \quad \xi \in \tilde{M}
$$

Proof. By Lemma $15, E_{0}(\xi, \cdot)$ belongs to $\operatorname{Exp}\left(\tilde{M} ;\left(0, L^{*}\right)\right)$. Therefore, as in the proof of Lemma 13, the right-hand side of (17) is finite and does not depend on $s$. 
Lemmas 15, 6, Corollary 9 and (2) imply

$$
\begin{aligned}
\int_{\tilde{M}} f(s \zeta) E_{0}(\xi, \bar{\zeta} / s) d \tilde{M}(\zeta) & =\sum_{k=0}^{\infty} 2^{k} N(k, n) \int_{M} f_{k}\left(\zeta^{\prime}\right)\left(\xi \cdot \bar{\zeta}^{\prime}\right)^{k} d M\left(\zeta^{\prime}\right) \\
& =\sum_{k=0}^{\infty} f_{k}(\xi)=f(\xi), \quad \xi \in \tilde{M} .
\end{aligned}
$$

Let $A \geq 0$ and $T \in \operatorname{Exp}^{\prime}\left(\tilde{M} ;\left(A, L^{*}\right)\right)$. We define the F-Poisson transform $\mathcal{M} T$ of $T$ by

$$
\mathcal{M} T(\zeta)=\left\langle T_{\xi}, E_{0}(\xi, \zeta)\right\rangle
$$

Because $E_{0}(\cdot, \zeta) \in \operatorname{Exp}\left(\tilde{M} ;\left(0, L^{*}\right)\right), \mathcal{M} T(\zeta)$ is well-defined. It is easy to see that $\mathcal{M} T$ is an entire function on $\tilde{M}$.

ThEOREM 17 The F-Poisson transformation $\mathcal{M}$ establishes the following topological linear isomorphisms:

$$
\begin{array}{ll}
\text { (i) } \mathcal{M}: \operatorname{Exp}^{\prime}\left(\tilde{M} ;\left(A, L^{*}\right)\right) \stackrel{\sim}{\longrightarrow} \operatorname{Exp}\left(\tilde{M} ;\left[1 / A, L^{*}\right]\right), \quad 0 \leq A<\infty, \\
\text { (ii) } \mathcal{M}: \operatorname{Exp}^{\prime}\left(\tilde{M} ;\left[A, L^{*}\right]\right) \stackrel{\sim}{\longrightarrow} \operatorname{Exp}\left(\tilde{M} ;\left(1 / A, L^{*}\right)\right), \quad 0<A \leq \infty .
\end{array}
$$

We have $\langle T, f\rangle=\langle\langle f, \mathcal{M} T\rangle\rangle_{\tilde{M}}$ for

$$
T \in \operatorname{Exp}^{\prime}\left(\tilde{M} ;\left(A, L^{*}\right)\right) \text { and } f \in \operatorname{Exp}\left(\tilde{M} ;\left(A, L^{*}\right)\right)
$$

or for

$$
T \in \operatorname{Exp}^{\prime}\left(\tilde{M} ;\left[A, L^{*}\right]\right) \text { and } f \in \operatorname{Exp}\left(\tilde{M} ;\left[A, L^{*}\right]\right) \text {. }
$$

Proof. We prove only (i). Let $T \in \operatorname{Exp}^{\prime}\left(\tilde{M} ;\left(A, L^{*}\right)\right)$. By the continuity of $T$, there are $A^{\prime}>A$ and $C \geq 0$ such that

$$
|\langle T, f\rangle| \leq C \sup \left\{|f(\xi)| \exp \left(-A^{\prime} L^{*}(\xi)\right) ; \xi \in \tilde{M}\right\} .
$$

Therefore, if $A<|s|<A^{\prime}$, then we have

$$
\begin{aligned}
|\mathcal{M} T(\zeta)| & \leq C \sup \left\{\exp \left(|s| L^{*}(\xi)+L^{*}(\zeta) /|s|\right) \exp \left(-A^{\prime} L^{*}(\xi)\right) ; \xi \in \tilde{M}\right\} \\
& \leq C \exp \left(L^{*}(\zeta) /|s|\right)
\end{aligned}
$$

that is, $\mathcal{M} T \in \operatorname{Exp}\left(\tilde{M} ;\left[1 / A, L^{*}\right]\right)$.

Let $f \in \operatorname{Exp}\left(\tilde{M} ;\left(A, L^{*}\right)\right)$. Take $s \in \mathbb{C}$ with $0<|s|<1 / A$ sufficiently close to $1 / A$. Then Theorem 16 implies

$$
\begin{aligned}
\langle T, f\rangle & =\left\langle T_{\xi}, \int_{\tilde{M}} f(s \zeta) E_{0}(\xi, \bar{\zeta} / s) d \tilde{M}(\zeta)\right\rangle \\
& =\int_{\tilde{M}} f(s \zeta)\left\langle T_{\xi}, E_{0}(\xi, \bar{\zeta} / s)\right\rangle d \tilde{M}(\zeta) \\
& =\int_{\tilde{M}} f(s \zeta) \mathcal{M} T(\bar{\zeta} / s) d \tilde{M}(\zeta)=\langle\langle f, \mathcal{M} T\rangle\rangle_{\tilde{M}}
\end{aligned}
$$

Thus, $\mathcal{M}$ is a continuous linear injection.

Conversely, let $F \in \operatorname{Exp}\left(\tilde{M} ;\left[1 / A, L^{*}\right]\right)$. Then $\left\langle T_{F}, f\right\rangle=\langle\langle f, F\rangle\rangle_{\tilde{M}}$ defines $T_{F} \in$ $\operatorname{Exp}^{\prime}\left(\tilde{M} ;\left(A, L^{*}\right)\right)$ and

$$
\mathcal{M} T_{F}(w)=\left\langle\left\langle E_{0}(z, w), F(z)\right\rangle\right\rangle_{\tilde{M}}=F(w)
$$


by Theorem 16 . Thus, $\mathcal{M}$ is surjective. The continuity of $\mathcal{M}^{-1}: F \mapsto T_{F}$ is clear.

6. The first main theorem. If $\zeta \in \tilde{B}(1 / R)$, then we have $\exp (\zeta \cdot z / \lambda) \in$ $\operatorname{Exp}\left(\tilde{M} ;\left[1 /(|\lambda| R), L^{*}\right]\right)$. Therefore, for $T \in \operatorname{Exp}^{\prime}\left(\tilde{M} ;\left[1 /(|\lambda| R), L^{*}\right]\right)$ we can define the Fourier-Borel transform

$$
\mathcal{F}_{1 / \lambda} T(\zeta)=\left\langle T_{z}, \exp (\zeta \cdot z / \lambda)\right\rangle, \quad \zeta \in \tilde{B}(1 / R) .
$$

It is clear that the Fourier-Borel transformation $\mathcal{F}_{1 / \lambda}$ is a continuous linear mapping

$$
\mathcal{F}_{1 / \lambda}: \operatorname{Exp}^{\prime}\left(\tilde{M} ;\left[1 /(|\lambda| R), L^{*}\right]\right) \longrightarrow \mathcal{O}_{\Delta}(\tilde{B}(1 / R))
$$

Taking inductive limit, we get another continuous linear mapping

$$
\mathcal{F}_{1 / \lambda}: \operatorname{Exp}^{\prime}\left(\tilde{M} ;\left(1 /(|\lambda| R), L^{*}\right)\right) \longrightarrow \mathcal{O}_{\Delta}(\tilde{B}[1 / R]) .
$$

We proved in [3] that (18) and (19) are topological linear isomorphisms. Here we claim that (18) and (19) give the inverse of the conical Fourier-Borel transformations (Lemma 12) via the isomorphisms $\mathcal{P}$ and $\mathcal{M}$. Thus, constructing explicitly the inverse mapping, we have another proof of the topological linear isomorphism of (18) and (19).

THEOREM 18. The following diagrams are commutative and all mappings are topological linear isomorphisms:

(i)

$$
\begin{array}{cccc}
\mathcal{O}_{\Delta}^{\prime}(\tilde{B}(R)) & \stackrel{\mathcal{P}^{-1}}{\longleftarrow} & \mathcal{O}_{\Delta}(\tilde{B}[1 / R]) & \\
\downarrow_{\mathcal{F}_{\lambda}^{\Delta}} & & \uparrow \mathcal{F}_{1 / \lambda} & (0<R \leq \infty) \\
\operatorname{Exp}\left(\tilde{M} ;\left[|\lambda| R, L^{*}\right]\right) & \stackrel{\mathcal{M}^{-1}}{\longrightarrow} & \operatorname{Exp}^{\prime}\left(\tilde{M} ;\left(1 /(|\lambda| R), L^{*}\right)\right), & \\
\mathcal{O}_{\Delta}^{\prime}(\tilde{B}[R]) & \stackrel{\mathcal{P}^{-1}}{\longleftarrow} & \mathcal{O}_{\Delta}(\tilde{B}(1 / R)) & (0 \leq R<\infty) \\
\downarrow_{\mathcal{F}_{\lambda}^{\Delta}} & & \uparrow \mathcal{F}_{1 / \lambda} & \\
\operatorname{Exp}\left(\tilde{M} ;\left(|\lambda| R, L^{*}\right)\right) & \stackrel{\mathcal{M}^{-1}}{\longrightarrow} & \operatorname{Exp}^{\prime}\left(\tilde{M} ;\left[1 /(|\lambda| R), L^{*}\right]\right) . &
\end{array}
$$$$
\text { (ii) }
$$

Proof. We prove only the first diagram. Because $\mathcal{P}$ and $\mathcal{M}$ are topological linear isomorphisms (Theorems 5 and 17), we have only to show that

$$
\text { 1) } \mathcal{F}_{\lambda}^{\Delta} \circ \mathcal{P}^{-1} \circ \mathcal{F}_{1 / \lambda} \circ \mathcal{M}^{-1}=\mathrm{id}, \quad \text { 2) } \mathcal{F}_{1 / \lambda} \circ \mathcal{M}^{-1} \circ \mathcal{F}_{\lambda}^{\Delta} \circ \mathcal{P}^{-1}=\mathrm{id}
$$

1) Let $f \in \operatorname{Exp}\left(\tilde{M} ;\left[|\lambda| R, L^{*}\right]\right)$. Then by Theorem 17 and Lemma 13, there is $s>$ $1 /(|\lambda| R)$ sufficiently close to $1 /(|\lambda| R)$ such that

$$
\mathcal{F}_{1 / \lambda}\left(\mathcal{M}^{-1} f\right)(z)=\int_{\tilde{M}} f(s \zeta) \exp (z \cdot \bar{\zeta} /(\lambda s)) d \tilde{M}(\zeta)
$$

Note that the right-hand side belongs to $\mathcal{O}_{\Delta}(\tilde{B}(s|\lambda|))$. By Theorem 5 , Lemma 4, the 
Fubini theorem, Lemma 15 and Theorem 16, there is $\rho$ with $1 / \rho<s|\lambda|$ such that

$$
\begin{aligned}
\left(\mathcal{F}_{\lambda}^{\Delta} \circ \mathcal{P}^{-1} \circ \mathcal{F}_{1 / \lambda} \circ \mathcal{M}^{-1}\right) f(\xi) \\
\quad=\int_{S} \mathcal{F}_{1 / \lambda}\left(\mathcal{M}^{-1} f\right)(\omega / \rho) \exp (\lambda \rho \omega \cdot \xi) d S(\omega) \\
\quad=\int_{S}\left(\int_{\tilde{M}} f(s \zeta) \exp (\omega \cdot \bar{\zeta} /(\lambda s \rho)) d \tilde{M}(\zeta)\right) \exp (\lambda \rho \omega \cdot \xi) d S(\omega) \\
\quad=\int_{\tilde{M}} f(s \zeta)\left(\int_{S} \exp (\omega \cdot \bar{\zeta} /(\lambda s \rho)) \exp (\lambda \rho \omega \cdot \xi) d S(\omega)\right) d \tilde{M}(\zeta) \\
\quad=\int_{\tilde{M}} f(s \zeta) E_{0}(\xi, \bar{\zeta} / s) d \tilde{M}(\zeta)=f(\xi), \quad \xi \in \tilde{M}
\end{aligned}
$$

2) Let $f \in \mathcal{O}_{\Delta}(\tilde{B}[1 / R])$. Then by Theorem 5 and Lemma 4 , there is $0<\rho<R$ such that

$$
\mathcal{F}_{\lambda}^{\Delta}\left(\mathcal{P}^{-1} f\right)(\zeta)=\int_{S} f(\omega / \rho) \exp (\lambda \rho \zeta \cdot \omega) d S(\omega), \quad \zeta \in \tilde{M}
$$

By Lemma $12, \mathcal{F}_{\lambda}^{\Delta}\left(\mathcal{P}^{-1} f\right)(\zeta)$ belongs to $\operatorname{Exp}\left(\tilde{M} ;\left[|\lambda| R, L^{*}\right]\right)$. Therefore, if $|s|=1 /(|\lambda| \rho)$, then Theorem 17, Lemma 13, the Fubini theorem, Lemma 14 and Theorem 2 imply

$$
\begin{aligned}
\left(\mathcal{F}_{1 / \lambda}\right. & \left.\circ \mathcal{M}^{-1} \circ \mathcal{F}_{\lambda}^{\Delta} \circ \mathcal{P}^{-1}\right) f(z) \\
& =\int_{\tilde{M}} \exp (z \cdot \zeta /(\lambda s)) \mathcal{F}_{\lambda}^{\Delta}\left(\mathcal{P}^{-1} f\right)(s \zeta) d \tilde{M}(\zeta) \\
& =\int_{\tilde{M}} \exp (z \cdot \zeta /(\lambda s))\left(\int_{S} f(\omega / \rho) \exp (\lambda \rho s \zeta \cdot \omega) d S(\omega)\right) d \tilde{M}(\zeta) \\
& =\int_{S} f(\omega / \rho)\left(\int_{\tilde{M}} \exp (z \cdot \zeta /(\lambda s)) \exp (\lambda \rho s \zeta \cdot \omega) d \tilde{M}(\zeta)\right) d S(\omega) \\
& =\int_{S} f(\omega / \rho) K_{1}(z, \rho \omega) d S(\omega)=f(z), \quad z \in \tilde{B}(1 / \rho) .
\end{aligned}
$$

7. Fourier-Borel transformation on $\mathcal{O}^{\prime}(\tilde{M}(R))$. In $[3]$ we studied the FourierBorel transformation on $\mathcal{O}^{\prime}(\tilde{M}(R))$. The Fourier-Borel transform $\mathcal{F}_{\lambda} T$ of $T \in \mathcal{O}^{\prime}(\tilde{M}(R))$ is defined by

$$
\mathcal{F}_{\lambda} T(z)=\left\langle T_{\zeta}, \exp (\lambda \zeta \cdot z)\right\rangle, \quad z \in \tilde{\mathbb{E}} .
$$

By the Hahn-Banach theorem, there is a Radon measure $\mu$ on $\tilde{M}(R)$ with

$$
\text { supp } \mu \subset \tilde{M}\left[R^{\prime}\right], \quad R^{\prime}<R,
$$

such that

$$
\mathcal{F}_{\lambda} T(z)=\int_{\tilde{M}\left(R^{\prime}\right)} \exp (\lambda \zeta \cdot z) d \mu(\zeta) .
$$

Therefore, $\mathcal{F}_{\lambda} T$ is an entire function on $\tilde{\mathbb{E}}$, satisfies $\Delta_{z}\left(\mathcal{F}_{\lambda} T\right)(z)=0$, and of exponential type:

$$
\begin{aligned}
\left|\mathcal{F}_{\lambda} T(z)\right| & \leq\|\mu\| \sup \left\{|\exp (\lambda \zeta \cdot z)| ; \zeta \in \tilde{M}\left[R^{\prime}\right]\right\} \\
& =\|\mu\| \exp \left(|\lambda| R^{\prime} L^{*}(z)\right) .
\end{aligned}
$$

To describe the image under $\mathcal{F}_{\lambda}$, let us introduce some notations. 
Let $N$ be the Lie norm $L$ or the dual Lie norm $L^{*}$. For $0<A \leq \infty$ we denote by

$\operatorname{Exp}(\tilde{\mathbb{E}} ;[A, N])=\left\{f \in \mathcal{O}(\tilde{\mathbb{E}}) ;\right.$ there is $A^{\prime}<A$ such that

$$
\left.\sup \left\{|f(z)| \exp \left(-A^{\prime} N(z)\right) ; z \in \tilde{\mathbb{E}}\right\}<\infty\right\}
$$

the space of entire functions on $\tilde{\mathbb{E}}$ of exponential type $[A, N]$. Put

$$
\operatorname{Exp}_{\Delta}(\tilde{\mathbb{E}} ;[A, N])=\operatorname{Exp}(\tilde{\mathbb{E}} ;[A, N]) \cap \mathcal{O}_{\Delta}(\tilde{\mathbb{E}}) .
$$

Furthermore, for $0 \leq A<\infty$ we put

$$
\begin{aligned}
\operatorname{Exp}(\tilde{\mathbb{E}} ;(A, N)) & =\left\{f \in \mathcal{O}(\tilde{\mathbb{E}}) ; \text { for all } A^{\prime}>A,\right. \\
& \left.\sup \left\{|f(z)| \exp \left(-A^{\prime} N(z)\right) ; z \in \tilde{\mathbb{E}}\right\}<\infty\right\}
\end{aligned}
$$

and $\operatorname{Exp}_{\Delta}(\tilde{\mathbb{E}} ;(A, N))=\operatorname{Exp}(\tilde{\mathbb{E}} ;(A, N)) \cap \mathcal{O}_{\Delta}(\tilde{\mathbb{E}})$.

Lemma 19. ([6, Proof of Corollary 3.5]) We have

$$
\begin{aligned}
& \operatorname{Exp}_{\Delta}\left(\tilde{\mathbb{E}} ;\left[A, L^{*}\right]\right)=\operatorname{Exp}_{\Delta}(\tilde{\mathbb{E}} ;[A / 2, L]), \\
& \operatorname{Exp}_{\Delta}\left(\tilde{\mathbb{E}} ;\left(A, L^{*}\right)\right)=\operatorname{Exp}_{\Delta}(\tilde{\mathbb{E}} ;(A / 2, L)) .
\end{aligned}
$$

In particular, if $f \in \operatorname{Exp}_{\Delta}\left(\tilde{\mathbb{E}} ;\left(A, L^{*}\right)\right)$, then for any $\epsilon>0$ there is $C \geq 0$ such that

$$
|f(x)| \leq C \exp ((A+\epsilon)\|x\| / 2), \quad x \in \mathbb{E} .
$$

A continuous linear functional on $\operatorname{Exp}_{\Delta}\left(\tilde{\mathbb{E}} ;\left[A, L^{*}\right]\right)$ or $\operatorname{Exp}_{\Delta}\left(\tilde{\mathbb{E}} ;\left(A, L^{*}\right)\right)$ will be called an entire harmonic functional on $\tilde{\mathbb{E}}$.

By the definition we have the following :

LEMMA 20. The Fourier-Borel transformation $\mathcal{F}_{\lambda}$ establishes the following continuous linear mappings:

$$
\begin{aligned}
& \mathcal{F}_{\lambda}: \mathcal{O}^{\prime}(\tilde{M}(R)) \longrightarrow \operatorname{Exp}_{\Delta}\left(\tilde{\mathbb{E}} ;\left[|\lambda| R, L^{*}\right]\right), \quad 0<R \leq \infty \\
& \mathcal{F}_{\lambda}: \mathcal{O}^{\prime}(\tilde{M}[R]) \longrightarrow \operatorname{Exp}_{\Delta}\left(\tilde{\mathbb{E}} ;\left(|\lambda| R, L^{*}\right)\right), \quad 0 \leq R<\infty .
\end{aligned}
$$

In [3] we proved that the transformations $\mathcal{F}_{\lambda}$ are topological linear isomorphisms by calculating the growth behavior of homogeneous components. Relying on the Plancherel measure on $\mathbb{E}$, we shall show that these isomophisms can be understood in the setting similar to Theorem 18.

8. The Plancherel measure on $\mathbb{E}$. On the Euclidean space $\mathbb{E}$ we introduce the Plancherel measure $d E$ by

$$
\int_{\mathbb{E}} f(x) d E(x)=\int_{0}^{\infty} \int_{S} f(r \omega) d S(\omega) r^{n-1} \rho_{n}(r) d r,
$$

where $\rho_{n}(r)$ is the Ii-Wada function defined by (1).

By means of the Plancherel measure on $\mathbb{E}$, we can define a duality bilinear form on $\operatorname{Exp}_{\Delta}\left(\tilde{\mathbb{E}} ;\left(A, L^{*}\right)\right) \times \operatorname{Exp}_{\Delta}\left(\tilde{\mathbb{E}} ;\left[1 / A, L^{*}\right]\right)$.

Lemma 21. Let $A \geq 0, f \in \operatorname{Exp}_{\Delta}\left(\tilde{\mathbb{E}} ;\left(A, L^{*}\right)\right)$ and $g \in \operatorname{Exp}_{\Delta}\left(\tilde{\mathbb{E}} ;\left[1 / A, L^{*}\right]\right)$.

1) The integral

$$
\int_{\mathbb{E}} f(s x) g(x / s) d E(x)
$$


converges absolutely for $s \in \mathbb{C}$ with $0<|s|<1 / A$ sufficiently close to $1 / A$.

2) The integral (20) is independent of $s$. The bilinear form

$$
\langle\langle f, g\rangle\rangle_{\mathbb{E}}=\int_{\mathbb{E}} f(s x) g(x / s) d E(x)
$$

is separately continuous. We have

$$
\langle\langle f, g\rangle\rangle_{\mathbb{E}}=\sum_{k=0}^{\infty} C(k, n) \int_{S} f_{k}(\omega) g_{k}(\omega) d S(\omega),
$$

where $f_{k}$ and $g_{k}$ are the $k$-harmonic components of $f$ and $g$, respectively and $C(k, n)$ are the constants defined by (2).

Proof. 1) By Lemma 19 there are $A^{\prime}>A$ and $C^{\prime} \geq 0$ such that

$$
|g(x / s)| \leq C^{\prime} \exp \left(\|x\| /\left(2|s| A^{\prime}\right)\right), \quad x \in \mathbb{E} .
$$

For any $A^{\prime \prime}$ with $A^{\prime}>A^{\prime \prime}>A$ there is $C^{\prime \prime} \geq 0$ such that

$$
|f(s x)| \leq C^{\prime \prime} \exp \left(\left(|s| A^{\prime \prime}\right)\|x\| / 2\right), \quad x \in \mathbb{E} .
$$

Therefore, for $|s|=1 / A^{\prime \prime}$ there is $C \geq 0$ such that

$$
|f(s x) g(x / s)| \leq C \exp \left(\left(1+A^{\prime \prime} / A^{\prime}\right)\|x\| / 2\right), \quad x \in \mathbb{E} .
$$

Because $\rho_{n}$ is of exponential type -1 , we have 1 ).

2) Take $s \in \mathbb{C} \backslash\{0\}$ as in 1). Then by Corollary 3, Lemma 1 and (2) we have

$$
\begin{aligned}
\int_{\mathbb{E}} f( & s x) g(x / s) d E(x) \\
& =\int_{0}^{\infty}\left(\int_{S} \sum_{k=0}^{\infty} s^{k} r^{k} f_{k}(\omega) \sum_{\ell=0}^{\infty} r^{\ell} s^{-\ell} g_{\ell}(\omega) d S(\omega)\right) r^{n-1} \rho_{n}(r) d r \\
& =\int_{0}^{\infty}\left(\sum_{k=0}^{\infty} r^{2 k} \int_{S} f_{k}(\omega) g_{k}(\omega) d S(\omega)\right) r^{n-1} \rho_{n}(r) d r \\
& =\sum_{k=0}^{\infty} C(k, n) \int_{S} f_{k}(\omega) g_{k}(\omega) d S(\omega) .
\end{aligned}
$$

Lemma 22. For $\xi, \zeta \in \tilde{M}$ with $L(\xi) L(\zeta)<1$ the Cauchy kernel $K_{0}(\xi, \zeta)$ can be represented as follows:

$$
K_{0}(\xi, \zeta)=\int_{\mathbb{E}} \exp (s \xi \cdot x) \exp (x \cdot \zeta / s) d E(x),
$$

where $s$ is sufficiently close to $1 / L(\xi)$.

Proof. The convergence of the integral can be proved as in the proof of Lemma 13. 
Now by Lemma 1, (2) and (9), we have

$$
\begin{aligned}
\int_{\mathbb{E}} \exp (s \xi \cdot x) \exp (x \cdot \zeta / s) d E(x) \\
=\sum_{k=0}^{\infty} C(k, n) \int_{S}\left((\xi \cdot \omega)^{k} / k !\right)\left((\zeta \cdot \omega)^{k} / k !\right) d S(\omega) \\
=\sum_{k=0}^{\infty} C(k, n) /\left((k !)^{2} N(k, n) \gamma(k, n)\right)(\xi \cdot \zeta)^{k} \\
=\sum_{k=0}^{\infty} 2^{k} N(k, n)(\xi \cdot \zeta)^{k}=K_{0}(\xi, \zeta)
\end{aligned}
$$

We define the F-Cauchy kernel $E_{1}(z, w)$ by

$$
E_{1}(z, w)=\int_{M} \exp (z \cdot \zeta) \exp (\bar{\zeta} \cdot w) d M(\zeta) .
$$

By the definition, $E_{1}(z, w)$ is a symmetric entire function on $\tilde{\mathbb{E}} \times \tilde{\mathbb{E}}$ and satisfies $\Delta_{z} E_{1}(z, w)$ $=\Delta_{w} E_{1}(z, w)=0$ and

$$
\left|E_{1}(z, w)\right| \leq \exp \left(L^{*}(z)+L^{*}(w)\right), \quad(z, w) \in \tilde{\mathbb{E}} \times \tilde{\mathbb{E}} .
$$

LEMMA 23. We have

$$
E_{1}(z, w)=\sum_{k=0}^{\infty} N(k, n) / C(k, n) \tilde{P}_{k, n}(z, w), \quad z, w \in \tilde{\mathbb{E}} .
$$

Especially, for any $s \in \mathbb{C} \backslash\{0\}$ we have

$$
E_{1}(s z, w / s)=E_{1}(z, w), \quad z, w \in \tilde{\mathbb{E}} .
$$

Proof. By (12), Lemma 6, (2) and (9), we have

$$
\begin{aligned}
E_{1}(z, w) & =\sum_{k=0}^{\infty} 1 /(k !)^{2} \int_{M}(z \cdot \zeta)^{k}(\bar{\zeta} \cdot w)^{k} d M(\zeta) \\
& =\sum_{k=0}^{\infty} 1 /\left((k !)^{2} 2^{k} N(k, n) \gamma(k, n)\right) \tilde{P}_{k, n}(z, w) \\
& =\sum_{k=0}^{\infty} N(k, n) / C(k, n) \tilde{P}_{k, n}(z, w) .
\end{aligned}
$$

The F-Cauchy kernel has a reproducing property in the following sense.

Theorem 24. Let $A \geq 0$ and $f \in \operatorname{Exp}_{\Delta}\left(\tilde{\mathbb{E}} ;\left(A, L^{*}\right)\right)$. For $s \in \mathbb{C}$ with $0<|s|<2 / A$ we have

$$
f(z)=\int_{\mathbb{E}} f(s x) E_{1}(z, x / s) d E(x), \quad z \in \tilde{\mathbb{E}} .
$$

Proof. By Lemma 23, $E_{1}(z, \cdot) \in \operatorname{Exp}_{\Delta}\left(\tilde{\mathbb{E}} ;\left(0, L^{*}\right)\right)$. Therefore, as in the proof of Lemma 21, the right-hand side of (21) is finite and independent of $s$. By Lemmas 21, 23, 
1 and Corollary 3, we have

$$
\begin{aligned}
\int_{\mathbb{E}} f(s x) E_{1}(z, x / s) d E(x) & =\sum_{k=0}^{\infty} N(k, n) \int_{S} f_{k}(\omega) \tilde{P}_{k, n}(z, \omega) d S(\omega) \\
& =\sum_{k=0}^{\infty} f_{k}(z)=f(z) .
\end{aligned}
$$

We define the F-Cauchy transform $\mathcal{E} T$ of $T \in \operatorname{Exp}_{\Delta}^{\prime}\left(\tilde{\mathbb{E}} ;\left(A, L^{*}\right)\right)$ by

$$
\mathcal{E} T(w)=\left\langle T_{z}, E_{1}(z, w)\right\rangle .
$$

Because $E_{1}(\cdot, w) \in \operatorname{Exp}_{\Delta}\left(\tilde{\mathbb{E}} ;\left(0, L^{*}\right)\right), \mathcal{E} T(w)$ is well-defined. It is easy to check that $\mathcal{E} T$ is an entire function on $\tilde{\mathbb{E}}$ and satisfies $\Delta_{w} \mathcal{E} T(w)=0$.

THEOREM 25. The F-Cauchy transformation $T \mapsto \mathcal{E} T$ establishes the following topological linear isomorphisms:

$$
\begin{array}{ll}
\mathcal{E}: \operatorname{Exp}_{\Delta}^{\prime}\left(\tilde{\mathbb{E}} ;\left(A, L^{*}\right)\right) \stackrel{\sim}{\longrightarrow} \operatorname{Exp}_{\Delta}\left(\tilde{\mathbb{E}} ;\left[1 / A, L^{*}\right]\right), & 0 \leq A<\infty, \\
\mathcal{E}: \operatorname{Exp}_{\Delta}^{\prime}\left(\tilde{\mathbb{E}} ;\left[A, L^{*}\right]\right) \stackrel{\sim}{\longrightarrow} \operatorname{Exp}_{\Delta}\left(\tilde{\mathbb{E}} ;\left(1 / A, L^{*}\right)\right), & 0<A \leq \infty .
\end{array}
$$

We have $\langle T, f\rangle=\langle\langle f, \mathcal{E} T\rangle\rangle_{\mathbb{E}}$ for

$$
T \in \operatorname{Exp}_{\Delta}^{\prime}\left(\tilde{\mathbb{E}} ;\left(A, L^{*}\right)\right) \text { and } f \in \operatorname{Exp}_{\Delta}\left(\tilde{\mathbb{E}} ;\left(A, L^{*}\right)\right)
$$

or for

$$
T \in \operatorname{Exp}_{\Delta}^{\prime}\left(\tilde{\mathbb{E}} ;\left[A, L^{*}\right]\right) \text { and } f \in \operatorname{Exp}_{\Delta}\left(\tilde{\mathbb{E}} ;\left[A, L^{*}\right]\right) .
$$

Proof. We prove only (i). Let $T \in \operatorname{Exp}_{\Delta}^{\prime}\left(\tilde{\mathbb{E}} ;\left(A, L^{*}\right)\right)$. By the continuity of $T$, there are $A^{\prime}>A$ and $C \geq 0$ such that

$$
|\langle T, f\rangle| \leq C \sup \left\{|f(z)| \exp \left(-A^{\prime} L^{*}(z)\right) ; z \in \tilde{\mathbb{E}}\right\}
$$

for any $f \in \operatorname{Exp}_{\Delta}\left(\tilde{\mathbb{E}} ;\left(A, L^{*}\right)\right)$. Therefore, if $A<|s|<A^{\prime}$, then we have

$$
\begin{aligned}
|\mathcal{E} T(w)| & \leq C \sup \left\{\exp \left(s L^{*}(z)+L^{*}(w) / s\right) \exp \left(-A^{\prime} L^{*}(z)\right) ; z \in \tilde{\mathbb{E}}\right\} \\
& \leq C \exp \left(L^{*}(w) / s\right) ;
\end{aligned}
$$

that is, $\mathcal{E} T \in \operatorname{Exp}_{\Delta}\left(\tilde{\mathbb{E}} ;\left[1 / A, L^{*}\right]\right)$.

Let $f \in \operatorname{Exp}_{\Delta}\left(\tilde{\mathbb{E}} ;\left(A, L^{*}\right)\right)$. Take $s \in \mathbb{C}$ with $0<|s|<1 / A$ sufficiently close to $1 / A$. Then Theorem 24 implies

$$
\begin{aligned}
\langle T, f\rangle & =\left\langle T_{z}, \int_{\mathbb{E}} f(s x) E_{1}(z, x / s) d E(x)\right\rangle \\
& =\int_{\mathbb{E}} f(s x)\left\langle T_{z}, E_{1}(z, x / s)\right\rangle d E(x) \\
& =\int_{\mathbb{E}} f(s x) \mathcal{E} T(x / s) d E(x)=\langle\langle f, \mathcal{E} T\rangle\rangle_{\mathbb{E}} .
\end{aligned}
$$

Thus $\mathcal{E}$ is a continuous linear injection.

Conversely, let $F \in \operatorname{Exp}_{\Delta}\left(\tilde{\mathbb{E}} ;\left[1 / A, L^{*}\right]\right)$. We define

$$
T_{F} \in \operatorname{Exp}_{\Delta}^{\prime}\left(\tilde{\mathbb{E}} ;\left(A, L^{*}\right)\right)
$$


by $\left\langle T_{F}, f\right\rangle=\langle\langle f, F\rangle\rangle_{\mathbb{E}}$ for $f \in \operatorname{Exp}\left(\tilde{\mathbb{E}} ;\left(A, L^{*}\right)\right)$. Then we have

$$
\mathcal{E} T_{F}(w)=\left\langle\left\langle E_{1}(x, w), F(x)\right\rangle\right\rangle_{\mathbb{E}}=F(w)
$$

by Theorem 24. Thus, $\mathcal{E}$ is surjective. The continuity of $\mathcal{E}^{-1}: F \mapsto T_{F}$ is clear.

9. The second main theorem. If $\zeta \in \tilde{M}(1 / R)$, then we have $\exp (\zeta \cdot z / \lambda) \in$ $\operatorname{Exp}_{\Delta}\left(\tilde{\mathbb{E}} ;\left[1 /(|\lambda| R), L^{*}\right]\right)$. Therefore, for $T \in \operatorname{Exp}_{\Delta}^{\prime}\left(\tilde{\mathbb{E}} ;\left[1 /(|\lambda| R), L^{*}\right]\right)$ we can define the conical Fourier-Borel transformation

$$
\mathcal{F}_{1 / \lambda}^{\Delta} T(\zeta)=\left\langle T_{z}, \exp (\zeta \cdot z / \lambda)\right\rangle, \quad \zeta \in \tilde{M}(1 / R) .
$$

It is clear that the conical Fourier-Borel transformation $\mathcal{F}_{1 / \lambda}^{\Delta}: T \mapsto \mathcal{F}_{1 / \lambda}^{\Delta} T$ is a continuous linear mapping

$$
\mathcal{F}_{1 / \lambda}^{\Delta}: \operatorname{Exp}_{\Delta}^{\prime}\left(\mathbb{E} ;\left[1 /(|\lambda| R), L^{*}\right]\right) \longrightarrow \mathcal{O}(\tilde{M}(1 / R)) .
$$

Taking inductive limit, we have another continuous linear mapping

$$
\mathcal{F}_{1 / \lambda}^{\Delta}: \operatorname{Exp}_{\Delta}^{\prime}\left(\mathbb{E} ;\left(1 /(|\lambda| R), L^{*}\right)\right) \longrightarrow \mathcal{O}(\tilde{M}[1 / R]) .
$$

We claim that these $\mathcal{F}_{1 / \lambda}^{\Delta}$ give the inverse of the Fourier-Borel transformations $\mathcal{F}_{\lambda}$ on $\mathcal{O}^{\prime}(\tilde{M}[R])$ and on $\mathcal{O}^{\prime}(\tilde{M}(R))$ via the isomorphisms $\mathcal{C}$ and $\mathcal{E}$.

Theorem 26. Let $\lambda \in \mathbb{C} \backslash\{0\}$. The following diagrams are commutative and all mappings are topological linear isomorphisms:

(i)

$$
\begin{array}{cccc}
\mathcal{O}^{\prime}(\tilde{M}(R)) & \left.\stackrel{\mathcal{C}^{-1}}{\mathcal{O}(\tilde{M}}[1 / R]\right) & \\
\downarrow_{\mathcal{F}_{\lambda}} & \uparrow_{\mathcal{F}_{1 / \lambda}^{\Delta}} & (0<R \leq \infty)
\end{array}
$$

$\operatorname{Exp}_{\Delta}\left(\tilde{\mathbb{E}} ;\left[|\lambda| R, L^{*}\right]\right) \stackrel{\mathcal{E}^{-1}}{\longrightarrow} \operatorname{Exp}_{\Delta}^{\prime}\left(\tilde{\mathbb{E}} ;\left(1 /(|\lambda| R), L^{*}\right)\right)$,

(ii)

$$
\begin{array}{cccc}
\mathcal{O}^{\prime}(\tilde{M}[R]) & \stackrel{\mathcal{C}^{-1}}{\longleftarrow} & \mathcal{O}(\tilde{M}(1 / R)) & \\
\downarrow \mathcal{F}_{\lambda} & & \uparrow \mathcal{F}_{1 / \lambda}^{\Delta} & (0 \leq R<\infty) \\
\operatorname{Exp}_{\Delta}\left(\tilde{\mathbb{E}} ;\left(|\lambda| R, L^{*}\right)\right) & \stackrel{\mathcal{E}^{-1}}{\longrightarrow} & \operatorname{Exp}_{\Delta}^{\prime}\left(\tilde{\mathbb{E}} ;\left[1 /(|\lambda| R), L^{*}\right]\right) . &
\end{array}
$$

P r o of. We prove only the first diagram. Since $\mathcal{C}$ and $\mathcal{E}$ are topological linear isomorphisms (Theorems 11 and 25), we have only to show that

$$
\text { 1) } \mathcal{F}_{\lambda} \circ \mathcal{C}^{-1} \circ \mathcal{F}_{1 / \lambda}^{\Delta} \circ \mathcal{E}^{-1}=\mathrm{id}, \quad \text { 2) } \mathcal{F}_{1 / \lambda}^{\Delta} \circ \mathcal{E}^{-1} \circ \mathcal{F}_{\lambda} \circ \mathcal{C}^{-1}=\text { id. }
$$

1) Let $f \in \operatorname{Exp}_{\Delta}\left(\tilde{\mathbb{E}} ;\left[|\lambda| R, L^{*}\right]\right)$. Then by Theorem 25 and Lemma 21, there is $s$ with $s>1 /(|\lambda| R)$ sufficiently close to $1 /(|\lambda| R)$ such that

$$
\mathcal{F}_{1 / \lambda}^{\Delta}\left(\mathcal{E}^{-1} f\right)(\zeta)=\int_{\mathbb{E}} f(s x) \exp (\zeta \cdot x /(\lambda s)) d E(x) .
$$

Note that the right-hand side belongs to $\mathcal{O}(\tilde{M}(s|\lambda|))$. Therefore, by Theorem 11, Lem- 
ma 10, the Fubini theorem and Theorem 24, there is $\rho$ with $1 / \rho<s|\lambda|$ such that

$$
\begin{aligned}
\left(\mathcal{F}_{\lambda}\right. & \left.\circ \mathcal{C}^{-1} \circ \mathcal{F}_{1 / \lambda}^{\Delta} \circ \mathcal{E}^{-1}\right) f(z) \\
& =\int_{M} \mathcal{F}_{1 / \lambda}^{\Delta}\left(\mathcal{E}^{-1} f\right)(\zeta / \rho) \exp (\lambda \rho \zeta \cdot z) d M(\zeta) \\
& =\int_{M}\left(\int_{\mathbb{E}} f(s x) \exp (\zeta \cdot x /(\lambda s \rho)) d E(x)\right) \exp (\lambda \rho \zeta \cdot z) d M(\zeta) \\
& =\int_{\mathbb{E}} f(s x)\left(\int_{M} \exp (\zeta \cdot x /(\lambda s \rho)) \exp (\lambda \rho \zeta \cdot z) d M(\zeta)\right) d E(x) \\
& =\int_{\mathbb{E}} f(s x) E_{1}(x / s, z) d E(x)=f(z), \quad z \in \tilde{\mathbb{E}} .
\end{aligned}
$$

2) Let $f \in \mathcal{O}(\tilde{M}[1 / R])$. Then by Theorem 11 and Lemma 10 , there is $\rho$ with $0<$ $\rho<R$ such that

$$
\mathcal{F}_{\lambda}\left(\mathcal{C}^{-1} f\right)(z)=\int_{M} f(\zeta / \rho) \exp (\lambda \rho z \cdot \bar{\zeta}) d M(\zeta), \quad z \in \tilde{\mathbb{E}} .
$$

From Lemma $20, \mathcal{F}_{\lambda}\left(\mathcal{C}^{-1} f\right)(z)$ belongs to $\operatorname{Exp}_{\Delta}\left(\tilde{\mathbb{E}} ;\left[|\lambda| R, L^{*}\right]\right)$. Therefore, for $s=$ $1 /(|\lambda| \rho)$, by Theorem 25, Lemma 21, the Fubini theorem, Lemma 22 and Theorem 8, we have

$$
\begin{aligned}
\left(\mathcal{F}_{1 / \lambda}^{\Delta} \circ \mathcal{E}^{-1} \circ \mathcal{F}_{\lambda} \circ \mathcal{C}^{-1}\right) f(\xi) \\
\quad=\int_{\mathbb{E}} \exp (x \cdot \xi /(\lambda s)) \mathcal{F}_{\lambda}\left(\mathcal{C}^{-1} f\right)(s x) d E(x) \\
\quad=\int_{\mathbb{E}} \exp (x \cdot \xi /(\lambda s))\left(\int_{M} f(\zeta / \rho) \exp (\lambda \rho s x \cdot \bar{\zeta}) d M(\zeta)\right) d E(x) \\
\quad=\int_{M} f(\zeta / \rho)\left(\int_{\mathbb{E}} \exp (x \cdot \xi /(\lambda s)) \exp (\lambda \rho s x \cdot \bar{\zeta}) d E(x)\right) d M(\zeta) \\
\quad=\int_{M} f(\zeta / \rho) K_{0}(\xi, \rho \bar{\zeta}) d M(\zeta)=f(\xi), \quad \xi \in \tilde{M}(1 / \rho) .
\end{aligned}
$$

\section{References}

[1] K. Ii, On a Bargmann-type transform and a Hilbert space of holomorphic functions, Tôhoku Math. J., 38 (1986), 57-69.

[2] M. Morimoto, Analytic functionals on the sphere and their Fourier-Borel transformations, in: Complex Analysis, Banach Center Publications 11, PWN-Polish Scientific Publishers, Warsaw, 1983, 223-250.

[3] M. Morimoto and K. Fujita, Analytic functionals and entire functionals on the complex light cone, to appear in Hiroshima Math. J., 25 (1995) or in 26 (1996).

[4] C. Müller, Spherical Harmonics, Lecture Notes in Math., 17 (1966), Springer.

[5] R. Wada, On the Fourier-Borel transformations of analytic functionals on the complex sphere, Tôhoku Math. J., 38 (1986), 417-432.

[6] R. Wada, Holomorphic functions on the complex sphere, Tokyo J. Math., 11 (1988), 205218.

[7] R. Wada and M. Morimoto, A uniqueness set for the differential operator $\Delta_{z}+\lambda^{2}$, Tokyo J. Math., 10 (1987), 93-105. 